\title{
Enhanced Bioactivity of Titanium by Laser-generated Lotus-Topographies: Molecular Insights in Osteogenic Signaling Pathways of hASCs
}

Fadeeva $\mathrm{E}^{1}$, Deiwick $\mathrm{A}^{1}$, Chichkov $\mathrm{B}^{1,2}$ and Schlie-Wolter $\mathbf{S}^{1,2 *}$

${ }^{1}$ Laser Zentrum Hannover e.V.; Nanotechnology Department; Hollerithallee 8; D-30419, Hannover, Germany

${ }^{2}$ Institute of Quantum Optics; Leibniz University Hannover; Welfengarten 1; D-30167, Hannover, Germany

\begin{abstract}
Laser-generated lotus-topographies were shown to fulfill important requirements of demanded bioactivity: inhibition of biofilms and fibrotic capsule formation and enhanced regeneration. Here, we figure out how the demonstrated markerspecific improved osteogenic differentiation of human adipose-derived stem cells (hASCs) is accomplished. Comparing flat and lotus-structured titanium, the role of the osteogenic signaling pathways via extracellular-signal related kinase (ERK), c-Jun-N-terminal kinase (JNK), and p38 mitogen-activated protein kinases (MAPKs), and interplay with ERK p-ERK and bone morphogenic protein (BMP2)/p-Smad signaling are determined. To our knowledge, this interaction has not been analyzed before. Blocking of ERK and JNK suppresses osteogenic markers like alkaline phosphatase (ALP) activity and calcium mineralization independently from the surface. Their functionality requires both transcription factors osterix and Runx2/p-Runx2, and interplay with ERK/p-ERK and BMP2/p-Smad. P38 plays a regulatory role enabling osteogenic differentiation, which depends on the surface design. Its inhibition generally increases ERK/p-ERK cascade resulting in a high extent of Runx2/p-Runx2. Surface dependent BMP2/p-Smad and osterix are activated. The chief cause in enhanced osteogenic differentiation correlates with the improved attachment on the lotus-topography. Therefore, laser-generated lotus-structures present an attractive surface functionalization, finding their application in diverse biomedical applications.
\end{abstract}

Keywords: Bioactivity; Titanium; Surface topography; Adiposederived stem cells; Osteoinduction

\section{Introduction}

Bioactivity can be seen as biological properties of implantable materials that go far beyond the well-known biocompatibility. Last includes non-toxic effects to the surrounding tissue and patient generally, which are evaluated following ISO 10993 before approving biomedical devices [1]. Unfortunately, long-time experiences in implantation medicine have demonstrated that biocompatibility is not sufficient to guaranty longevity and functionality of implants - causing harm to the patients and re-implantations with increasing health care costs. Implant failure can be caused by technical aspects such as fatigue, corrosion, and others [2]. But in the past it became apparent that biological problems as infections caused by biofilms and fibrotic capsule formation related to immune rejection are intractable. Up to now these biological problems could not be getting under control - and there is a long-life risk for the patients to suffer from them.

Coming back to term 'bioactivity', it means that the biomaterial is not passive; tissue-implant interactions do not occur randomly; the biomaterial can directly interfere with the tissue; it shall actively control tissue responses in the way it is needed. In the first place this control deals with an enhanced regeneration of the surrounding tissue [3]. This is classified by cell anchorage and differentiation. In the second place biofilm and fibrotic capsule formation shall be inhibited from the very beginning [4,5]. Therefore, a selective cell control is required [6]. Many efforts have been undertaken to test which material properties can enhance the bioactivity. The current strategy is based on changing the material surface $[2,6-8]$. Beside the technical challenge to modify it in a reproducible manner, the big question is what kind of surface characteristics are demanded for the certain application. For good outcome, the natural environment of cells should be mimicked. To form a concept, properties of the cell-surrounding extracellular matrix (ECM) should be copied. This ECM represents defined mechanical features, certain geometry, specific anchorage points for cell-binding and further storages signaling molecules like growth factors cells require for living [9]. These so-called functionalization approaches have a physical or biochemical nature. First is characterized by changes in mechanics, wettability, and charges as well as topological features. Last incorporates a surface design with cell anchorage points such as adhesion ligands or adhesive peptides and a controlled delivery of signaling molecules. In the past many studies have demonstrated that functionalization of biomaterial surfaces enhances the bioactivity $[6,10-12]$. Looking at the literature it can be criticized that the studies are difficult to be compared due to the non-standardized applied protocols and investigated cell type diversity. Most of the work was not concentrated on all important aspects of bioactivity - regeneration, infection plus immune response, and in vitro and in vivo assays were rarely performed in parallel. About 10 years of study on the functionality of laser-generated surface topographies, an enhanced bioactivity could widely be demonstrated by us: (a) influence on biofilm formation, (b) inhibition of fibrotic cells in vitro and in vivo, (c) improved cell anchorage and differentiation for neuronal and orthopedic applications [6,13-17]. Attractive technical aspects of laserbased biomaterial functionalization refer to the high reproducibility of defined surface designs in micro- and nano-scale without negative influence on the biocompatibility of the used material.

Concerning osteogenic differentiation, we observed that not all

*Corresponding author: Sabrina Schlie-Wolter, Laser Zentrum Hannover e.V. Hollerithallee 8, 30419 Hannover, Germany, Tel: +49-511-2788-303; Fax: +49-5112788-100; E-mail: s.schlie@Izh.de

Received October 18, 2016; Accepted October 24, 2016; Published October 28 2016

Citation: Fadeeva E, Deiwick A, Chichkov B, Schlie-Wolter S (2016) Enhanced Bioactivity of Titanium by Laser-generated Lotus-Topographies: Molecular Insights in Osteogenic Signaling Pathways of hASCs. J Nanomed Nanotechnol 7: 403. doi: 10.4172/2157-7439.1000403

Copyright: ( $) 2016$ Fadeeva E, et al. This is an open-access article distributed under the terms of the Creative Commons Attribution License, which permits unrestricted use, distribution, and reproduction in any medium, provided the original author and source are credited. 
typical osteogenic markers were increased by a lotus-topography in titanium (Ti) [17]. This indicates that the osteogenic signaling pathways differ for each marker and/or the topography can selectively interfere on the molecular level. Both theories are not far-fetched looking at the multiple, interconnected and still unknown possibilities how the osteogenic transcription factors being responsible for the osteogenic markers can become activated [18]. Since the role of signaling cascades cannot be generalized due to cell specific differences, results can further correlate with the applied method [19], research is required to identify the role of the surface functionalization. This research is absolutely necessary to guaranty the effectiveness of the bioactivity. Above that knowledge on the cell biological details of biomaterial-cell interactions could facilitate future functionalization approaches.

Here, we are interested in the osteogenic molecular signaling pathways in dependence of extracellular-signal related kinase (ERK), c-Jun-N-terminal kinase (JNK), and p38 mitogen-activated protein kinases (MAPKs). Additionally, the interplay with ERK/p-ERK and bone morphogenic protein (BMP2)/pSmad pathway is determined. All of them were shown to be involved in osteogenic differentiation [18]. Their impact on osteogenic differentiation of human adipose derived stem cells (hASCs) is estimated via the osteogenic transcription factors osterix, Runx2/p-Runx2 following the activation of osteogenic markers such as alkaline phosphatase (ALP) activity and calcium mineralization; and the impact on proliferation and actin cytoskeleton with focal adhesion complex formation (via phosphorylated focal adhesion kinase (p-FAK Tyr397)). The main focus is finding out the influence on these cascades by the Ti lotus-topography.

\section{Materials and Methods}

\section{Surface structuring}

Lotus-topographies were generated on disk shaped titanium samples (Goodfellow $\mathrm{GmbH}$ ) with $6 \mathrm{~mm}$ in diameter and $0.25 \mathrm{~mm}$ in thickness. For surface structuring, a commercially available amplified Ti-Sapphire femtosecond laser system (Femtopower Compact Pro, Femtolasers Produktions GmbH, Vienna, Austria) was used. It delivers sub-30-fs pulses at $800 \mathrm{~nm}$ wavelength with energy of up to $1 \mathrm{~mJ}$ and a repetition rate of $1 \mathrm{kHz}$. An achromatic lens with a focal distance of 200 $\mathrm{mm}$ was used for focusing the laser beam. The laser beam was scanned along its linear polarization direction ( $\mathrm{x}$-direction). The scanning speed in the $\mathrm{x}$-direction was $800 \mu \mathrm{m} / \mathrm{s}$ and the translation step along the $y$-direction was $15 \mu \mathrm{m}$. Lotus-topographies were generated by laser ablation at a fluence of $8 \mathrm{~J} / \mathrm{cm}^{2}$ by using of circularly polarized laser light [17]. For the cell studies, both Ti flat and lotus-topographies were sterilized under UV light for $30 \mathrm{~min}$.

\section{Cell culture and experimental setup}

All chemicals were purchased from Sigma-Aldrich (München, Germany) unless otherwise noted. Human adipose-derived stem cells (hASCs), used as passage 4-5, were purchased from Lonza (Basel, Switzerland; Lot number 0000399065). The original control medium Dulbecco's modified Eagles medium, including 10\% foetal bovine serum (Biochrom AG, Berlin, Germany) and antibiotics, was additionally supplemented with $0.1 \mu \mathrm{M}$ dexamethasone, 10 $\mathrm{mM} \beta$-glycerolphosphate, and $50 \mu \mathrm{M}$ ascorbate-2-phosphate to induce osteogenic differentiation. To block the enzymes involved in osteogenic differentiation, following inhibitors and concentration were added to the osteogenic culture media: $20 \mu \mathrm{M}$ ERK (U0126, Biomol, Hamburg, Germany); $30 \mu \mathrm{M}$ JNK (SP600125, Santa Cruz Biotechnology, Heidelberg, Germany), $10 \mu \mathrm{M}$ p38 (SB203580, Abcam,
Cambridge, UK). Since the inhibitors were prepared in dimethyl sulfoxide (DMSO), a DMSO solvent control with a concentration of $0.04 \% \mathrm{v} / \mathrm{v}$ in osteogenic media was additionally tested. The media was changed every third day. For each experiment $1.8 \times 10^{4} \mathrm{hASCs}$ per treatment was seeded out into 48 well plates filled with $300 \mu \mathrm{l}$ cell culture media. Staining analysis of the actin cytoskeleton, p-FAK Tyr ${ }^{397}$ as a component of focal adhesion complexes, the transcription factors osterix, Runx2/p-Runx2, ERK/-p-ERK and BMP2/-p-Smad signaling was performed after 3 days cultivation time; proliferation, ALP activity, and calcium mineralization after 7, 14, and 21 days.

The osteogenic signaling cascades were analyzed on the control surface (well plate bottom) first. For this purpose, undifferentiated hASCs were seeded out in control medium, osteogenic medium, and osteogenic medium supplemented with DMSO solvent and each inhibitor, respectively. Since control hASCs did not undergo osteogenic differentiation as shown for the markers ALP activity and calcium mineralization and significantly reduced osteogenic transcription factors (Supplementary Figure 1) [17,18], they were not used for the further studies on the comparison of Ti flat and Ti lotus. Following the staining protocols, the impact of p38 on osteogenic hASCs cultivated on the control surface was additionally determined (Supplementary Table 1), whereas the influences of ERK and JNK on the control surface can be found in [18].

\section{Imaging and staining protocols}

Imaging was performed after 3 days cultivation time. To document cell morphology and adhesion in dependence of the surface and inhibitors, scanning electron microscopy (Microscope Quanta 400F ESEM (FEI Company, Oregon, USA)) was used. After washing the samples with phosphate buffer saline (PBS), they were fixed with $2.5 \%$ glutaraldehyde for $15 \mathrm{~min}$, followed by $2 \%$ osmium tetroxid for $30 \mathrm{~min}$. After washing steps and a dehydration series with increasing ethanol concentrations, the samples incubated in hexamethyldisilazane. Finally, the samples were sputter-coated with gold and visualized [17].

For molecular insights, the cells were fixed in $4 \%$ paraformaldehyde and permeabilized with $0.3 \%$ Triton X-100/PBS. To prevent nonspecific antibody binding, a $2 \%$ bovine serum albumin/PBS solution was incubated at $37^{\circ} \mathrm{C}$ for $2 \mathrm{~h}$. Afterwards, the cells were kept in the primary antibody solution, diluted in $0.3 \%$ Triton X-100/PBS, at $4^{\circ} \mathrm{C}$ overnight. Following primary antibodies and concentrations were applied: p-FAK (rabbit, 1:200; Cell Signaling, Danvers, USA), osterix (rabbit, 1:50, Santa Cruz Biotechnology), Runx2 (goat, 1:50, Santa Cruz Biotechnology, Heidelberg, Germany), p-Runx2 (rabbit, 1:100, Bioss, Freiburg, Germany), ERK (rabbit, 1:100, Cell Signaling), p-ERK (rabbit, 1:200, Cell Signaling), BMP2 (goat, 1:50, Santa Cruz Biotechnology), p-Smad 1/5/8 (rabbit, 1:200, Cell Signaling). After several washing steps, the fluorescence-conjugated secondary antibody was added at $37^{\circ} \mathrm{C}$ for $1 \mathrm{~h}$ (Alexa Fluor ${ }^{\oplus} 488$ conjugated Goat anti-rabbit IgG $(\mathrm{H}+\mathrm{L}) /$ Alexa Fluor $^{\circledR}$ 555 conjugated Goat anti-rabbit IgG $(\mathrm{H}+\mathrm{L})$, Dianova, Hamburg, Germany). Cell nuclei were stained with Hoechst 33342. For staining of actin filaments, phalloidin-Atto 550 was added for $20 \mathrm{~min}$. The cells were kept in PBS for further imaging using a fluorescence microscope (Nikon Eclipse, TE 2000-E, Nikon, Düsseldorf, Germany). All pictures were taken with the same image recordings.

To quantify the relative fluorescence intensities, ImageJ software was applied (http://rsbweb.nih.gov/ij). For the transcription factors and p-Smad, a rectangle of $2.5 \times 10^{3}$ pixels was placed on the nuclei, for ERK, p-ERK and BMP2 staining a rectangle with 10x103 pixels was placed on the entire cell bodies. For each image the corresponding rectangle 
size was placed aside the cells to estimate the background signal. Using the task 'measure RGB', the color intensity could automatically be quantified. Thereafter, the corresponding background was subtracted. These relative results were averaged \pm SEM and obtained from 100 rectangles per treatment [18].

\section{Proliferation and quantification of osteogenic differentiation}

Both analyses were performed with cell lysis, which were obtained after washing the samples with PBS and treatment with $1 \%$ Triton X-100/PBS [18]. The cell lysis was centrifuged at 12,000 g for $10 \mathrm{~min}$, whereupon the supernatant was used to quantify the cell density for proliferation and osteogenic marker ALP activity. The pellet and bottom of the sample were incubated with $0.5 \mathrm{~N} \mathrm{HCl}$ at $4^{\circ} \mathrm{C}$ overnight. Afterwards, both solutions for each sample were combined and utilized to measure calcium content as an additional osteogenic marker. To quantify the absorbance for each value, a microplate reader was used (Tecan Infinite M200Pro with i-controlTM software, Crailsheim, Germany). All results were obtained of three independent measurements after subtracting the blank and given as average \pm SEM.

Following the online protocol of OPS Diagnostics (Lebanon, USA), the cell density was measured via lactate dehydrogenase (LDH) assay. The LDH activity was detected at $492 \mathrm{~nm}$ wavelength and correlated with a standard cell curve prepared under the same conditions. The results were expressed as cell number $/ \mathrm{cm}^{2}$.

The ALP activity was correlated with a standard curve of p-nitrophenol ( $10 \mathrm{mmol} / \mathrm{L})$. Using $405 \mathrm{~nm}$ wavelengths, the ALP activity results of each sample were represented as nmol of p-nitrophenol/min/ $\mathrm{cm}^{2}$. Measuring calcium content followed the protocol of liquicolor assay (Human Diagnostics, Wiesbaden, Germany). Here, the standard calcium curve was raised from a stocking solution of $8 \mathrm{mg} / \mathrm{dL}$ calcium. Calcium was measured at $570 \mathrm{~nm}$ wavelength and given as $\mu \mathrm{g} / \mathrm{cm}^{2}$.

\section{Statistical analysis}

To analyze statistical differences, an unpaired two-sample Student's-t-test was applied with significant levels of $p<0.05\left(^{*}\right), p<0.01$ $\left({ }^{* *}\right)$, and $\left.\mathrm{p}<0.001{ }^{* * *}\right)$. First, DMSO solvent and applied inhibitors for ERK, JNK, and p38 were compared with the osteogenic setup $\left(^{*}\right)$; second, Ti flat with lotus-topography (\#).

\section{Results and Discussion}

Many studies have demonstrated that a biochemical and physical functionalization of biomaterial surfaces enhances differentiation of stem cells [6,20-22]. This observation can be transferred to biomedicine in a way that such surface designs might improve tissue regeneration after implantation - one criterion of enhanced material bioactivity. An attractive surface modification refers to laser-structuring. It enables the precise and reproducible manufacturing of topologies in microand nano-scale and enhances the demanded bioactivity: inhibition of biofilm and fibrotic capsule formation, improvement of neuronal and osteogenic differentiation [6,13-17]. However, in what manner material properties improve differentiation generally, how the specific material information are transferred to cells and stimulate molecular signaling pathways, requires further cell biological analysis in terms of biomaterial-cell interactions.

For orthopedic applications several cell types and markers are taken into account to classify osteogenic differentiation. Human mesenchymal stem cells derived from adipose tissue (hASCs) became an attractive cell source in the past, due to the abundant supply after routine liposuction, immunosuppressive properties, and differentiation efficiency in vitro and in vivo $[18,23]$. In that connection it has to be pointed out that all analyzed osteogenic markers, their extent and duration, depend on the cell type, donor, and applied method [19]. Therefore, obtained results cannot be generalized.

Osteogenic markers of interest are ALP activity, calcium mineralization and specific osteogenic proteins such as osteocalcin or osteopontin [24]. The progress of ALP activity is characterized by an increase up to a maximum followed by a decrease. For hASCs this maximum occurred after 14 days cultivation time (Figure 4 and Supplementary Figure 1B). Since hASCs cultivated with control cell medium did not present these markers on control surfaces and functionalized $\mathrm{Ti}$, they were not further analyzed (Supplementary Figure $1 \mathrm{~B}$ and 1C) [17]. Concerning the enhanced osteogenic differentiation of hASCs on laser-generated lotus-topographies in $\mathrm{Ti}$, we concluded that this improvement correlated with a control of cell adhesion mechanism on the lotus-structures: a different localization of integrin receptors occurred, hASCs attached significantly stronger, which was measured via adhesion forces, with a higher degree of vinculin as part of focal adhesion complexes [17]. Here, we additionally demonstrated that the cells were elongated on the substrates, stretching over many micro-sized surface features and forming diverse cellular extensions being in contact with the nano-roughness (Figure 1). Even though the cells were less expanded and reduced stress fibers on the lotus-structures compared with $\mathrm{Ti}$ flat, it supports the finding that the cells attached - since p-FAK was basically expressed at the end of the stress fibers, it indicates the formation of focal adhesions (Figure 2). For osteogenic cells higher cell densities on the control surface occurred, while on Ti flat and lotus it was comparable; therefore, no clear surface structure effect occurred (Figure 3 and Supplementary Figure 1A).

On the lotus-topography marker specific effects were estimated. For osteogenic hASCs ALP activity was significantly increased; but calcium values were similar (Figure 4). This is in contradiction to our first study, where the results were opposite [17]. Since different analyses were applied, it supports the fact that discrepancies can be raised from the method $[18,19]$.

Generally, the mentioned osteogenic differentiation markers are activated by the transcription factors osterix and Runx2 [18]. Their transcriptional activation requires cofactors and phosphorylation, which in turn is initiated by diverse molecular signaling cascades and interplays [25]. In this connection a regulation via MAPK cascades (via JNK, ERK, and p38) and BMP-Smad pathway are suggested [18] Comparing it with the control surface, it turned out that Ti flat and lotus significantly increased Runx2 and p-Runx2; osterix was also significantly increased on $\mathrm{Ti}$ flat, but similar values on Ti lotus were obtained (Figure 5 and Table 1B). Thereby, osterix and Runx2 were basically located within the nuclei, while $\mathrm{p}$-Runx 2 was further found

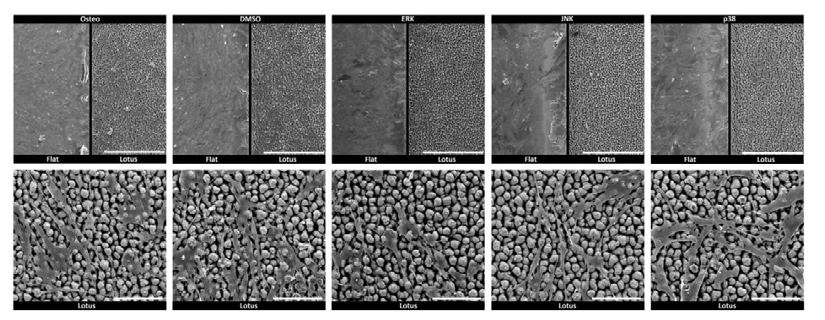

Figure 1: Scanning electron microscope images of osteogenic hASCs cultivated with the DMSO solvent control and different inhibitors after 3 days cultivation time. Scale bars: overview image $=500 \mu \mathrm{m}$, detail image $=100 \mu \mathrm{m}$. 
Citation: Fadeeva E, Deiwick A, Chichkov B, Schlie-Wolter S (2016) Enhanced Bioactivity of Titanium by Laser-generated Lotus-Topographies: Molecular Insights in Osteogenic Signaling Pathways of hASCs. J Nanomed Nanotechnol 7: 403. doi: 10.4172/2157-7439.1000403

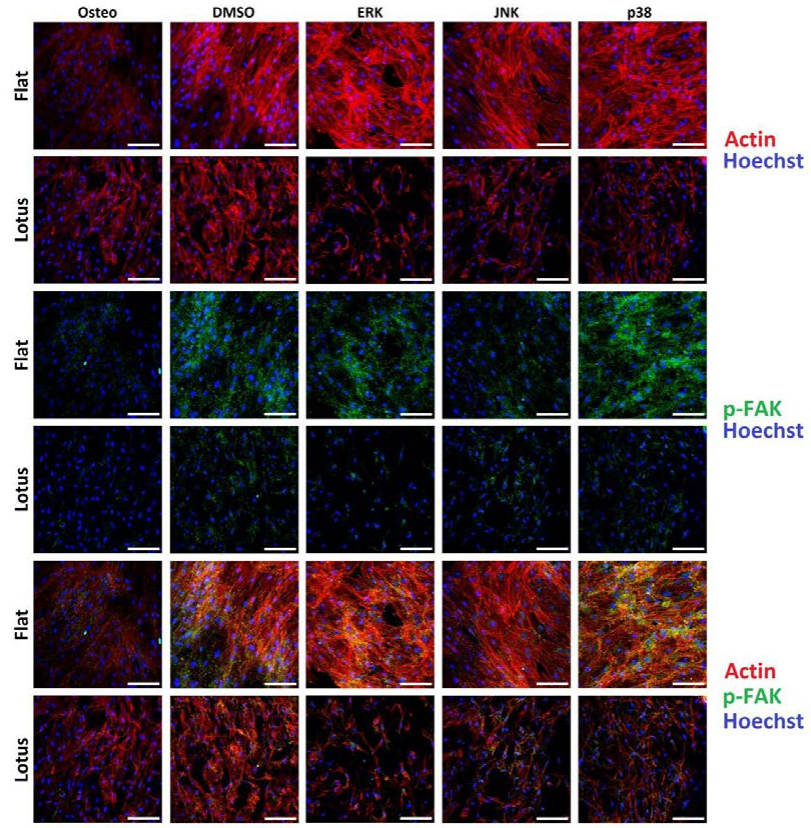

Figure 2: Fluorescence images of actin cytoskeleton (red) and p-FAK Tyr ${ }^{397}$ (green) as part of focal adhesion complex after 3 days cultivation time. Nuclei (blue) are stained via Hoechst. Scale bars $=100 \mu \mathrm{m}$
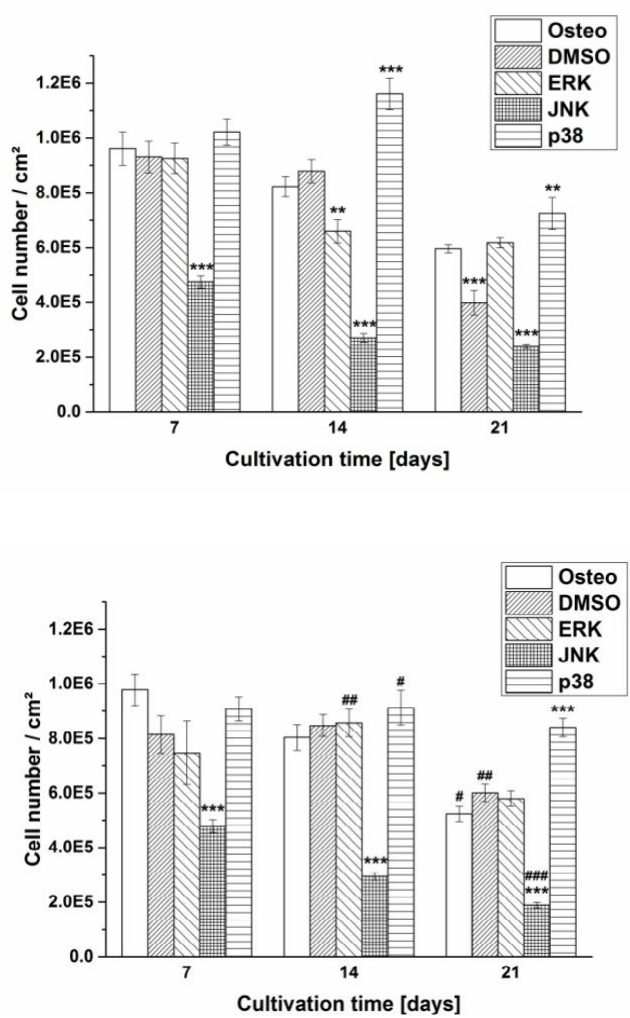

Figure 3: Proliferation profiles estimated via LDH assay. The averaged results [cell number $\left./ \mathrm{cm}^{2}\right]+\mathrm{SEM}$ were obtained from three independent measurements of $(A)$ Ti flat and (B) lotus topography. An average cell density of $1.8 \times 10^{4}$ cells per treatment was seeded out. Student's-t-test was applied $(p<0.05, p<0.01$, $p<0.001$ ) to estimate significant differences between osteogenic and DMSO setup, and effects of the inhibitors of ERK, JNK, and p38 (*); and Ti flat with lotus topography (\#) in the entire cell bodies after three days (Figure 5A and 5C). It can be concluded that both transcription factors are involved in osteogenic differentiation, which supports previous studies [18]. The higher extent might indicate an improvement of differentiation, whereas Runx2 signaling was more dominant on the lotus-topography. An increase of Runx2 expression was also documented for MSCs cultivated on surface-structured $\mathrm{Ti}$ and zirconia [26]. However, the activation mechanism of the transcription factor remained unclear.

Focusing on the interplay with ERK/p-ERK and BMP2/p-Smad signaling, surface specific influences for hASCs were estimated (Figures 6, 7 and Table 1B). ERK, p-ERK, BMP2, and p-Smad were located over the entire cell bodies; whereas p-Smad was dominantly presented within the nuclei after 3 days in culture. Control hASCs significantly reduced the amount for ERK, p-ERK, and p-Smad on the control surface, while the BMP2 value was comparable with the osteogenic cells (Table 1) [18]. Osteogenic hASCs significantly increased ERK/ p-ERK on Ti flat; the activated form p-ERK on Ti lotus as well. This indicates that osteogenic differentiation Ti flat and lotus was dependent on interplay with this pathway. BMP2 values were either similar on $\mathrm{Ti}$ flat or significantly reduced on Ti lotus, while p-Smad was significantly increased on Ti flat or comparable with lotus topography. This can be interpreted in a way that the BMP2/p-Smad pathway is involved in osteogenic differentiation on Ti flat - but less important on Ti lotus. Generally, the role of BMP2/p-Smad is not solved for hASCs [18]. These findings indicate that this pathway correlates with the external cell environment, but further research is necessary to classify the interplay.

To get more insights in the MAPK cascade, ERK, JNK, and p38 were specifically blocked. Additionally, a DMSO solvent control was tested, which caused no remarkable impacts. JNK inhibition resulted in a changed cell morphology and poor focal adhesion formation; additionally cell proliferation was significantly reduced. ERK and p38 not clearly affected adhesion and morphology, while p38 significantly increased cell growth and ERK had no impact (Figures 1-3 and Supplementary Figure 1A) [18].

Starting with the osteogenic markers, specific effects occurred (Figure 4 and Table 1). Inhibition of JNK and ERK significantly reduced ALP activity (day 14) and calcium production (day 21) on all surfaces when compared with osteogenic hASCs, which supports the findings in [18]. The maximum of ALP activity was delayed to day 21, but still lower rates than for osteogenic cells were measured. The ALP values were lower than on the control surface, whereas the decrease was less on Ti lotus. On Ti flat calcium mineralization was higher for both inhibitors than on the control; on Ti lotus ERK blocking reduced it and JNK increased it. Thereby, the lotus-topography slightly supported ALP activity, but not calcium mineralization. It can be concluded that JNK and ERK are the main candidates to promote osteogenic differentiation independently from the surface. With respect to osteogenic hASCs, inhibition of p38 was more dependent on the surface: ALP activity was similar on the control surface, significantly increased on $\mathrm{Ti}$ flat and reduced on Ti lotus; calcium mineralization was higher on the control surface and Ti flat, but comparable on Ti lotus. With respect to the surface design, the ALP activity was similar on the control surface and Ti flat, while the lotus-topography strongly reduced it. Similar to ERK and JNK blocking, the ALP activity maximum was delayed to day 21 . Here, high rates on Ti lotus occurred, which were comparable with osteogenic hASCs. Calcium mineralization significantly followed the order: control surface $<$ Ti lotus $<$ Ti flat. Since blocking of p 38 did not abolish the investigated markers can denote that this MAPK cascade 
Citation: Fadeeva E, Deiwick A, Chichkov B, Schlie-Wolter S (2016) Enhanced Bioactivity of Titanium by Laser-generated Lotus-Topographies: Molecular Insights in Osteogenic Signaling Pathways of hASCs. J Nanomed Nanotechnol 7: 403. doi: 10.4172/2157-7439.1000403

Page 5 of 9

A

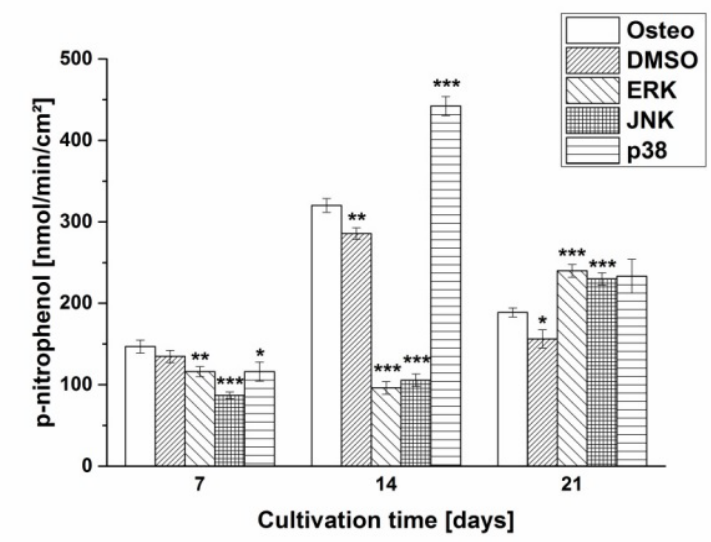

C

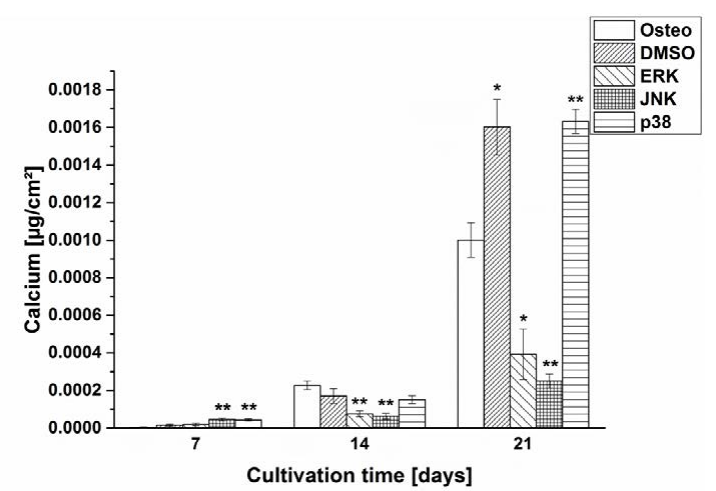

B

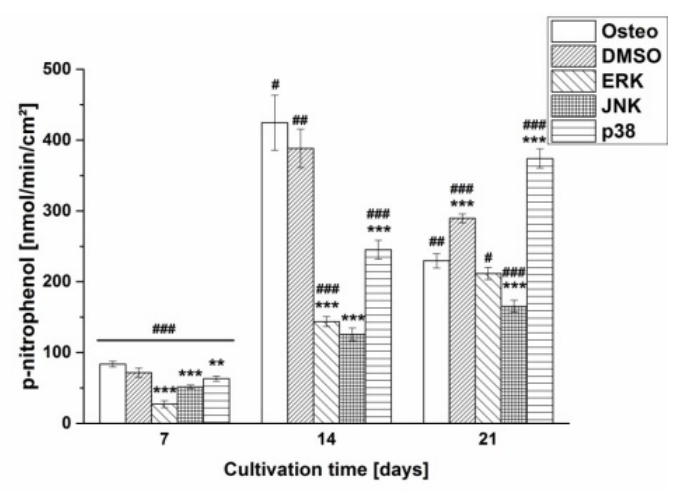

D

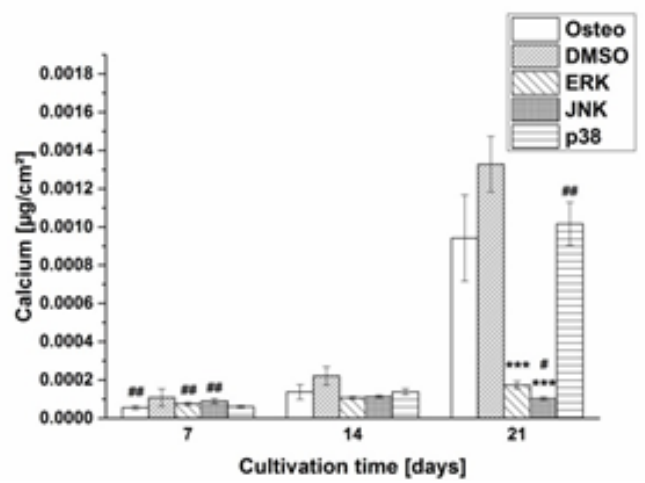

Figure 4: Quantification of the osteogenic markers at different time points of cultivation, comparing Ti flat (A, C) and lotus topography (B, D). (A, B) ALP activity via p- nitrophenol $\left[\mathrm{nmol} / \mathrm{min} / \mathrm{cm}^{2}\right]$; (C, D) calcium mineralization $\left[\mu \mathrm{g} / \mathrm{cm}^{2}\right]$. The averaged results \pm SEM were obtained of three independent measurements. An average cell density of $1.8 \times 10^{4}$ cells per treatment was seeded out. Student's-t-test was applied ( $\left.p<0.05, p<0.01, p<0.001\right)$ to estimate significant differences between osteogenic and DMSO setup, and effects of the inhibitors of ERK, JNK, and p38 ( $\left.{ }^{*}\right)$; and Ti flat with lotus topography (\#).

component does not play a role in osteogenic differentiation - or its inhibition activates other signaling pathways that are linked to it in order to sustain lineage commitment. Other studies also assumed that p38 plays a negative role in osteoblast differentiation [27]. However, the significant differences between the surfaces indicate that the functionality of p38 strongly correlates with the external cell environment.

On the control surface, blocking of ERK and JNK significantly reduced osterix and Runx2/p-Runx2, when compared with osteogenic hASCs. This means that they require both transcription factors for osteogenic differentiation [18]. Inhibition of p38 also blocked osterix, but Runx 2 values were comparable and p-Runx 2 was even significantly increased. The impact on osterix was not a novelty, since p38 directly activates osterix [25]. It can be concluded that the sustained differentiation after p38 blocking only correlated with Runx2/p-Runx2 pathway. The presence of ERK and JNK inhibitors further suppressed osterix on Ti flat and lotus. On Ti flat Runx2 was not affected by ERK and JNK, while p-Runx 2 was significantly increased after ERK and reduced after JNK treatment. On Ti lotus ERK reduced Runx2/pRunx2, while JNK only reduced p-Runx2. Even though both inhibitors reduced the transcription factors on Ti less than on the control surface - except for osterix on Ti lotus, the suppression correlated with the observed inhibition of osteogenic markers. The sustained p-Runx2 expression after ERK treatment was not sufficient for osteogenic differentiation, indicating that osteogenic lineage commitment of these MAPKs requires both transcription factors. Similar to the osteogenic markers, the impact of $\mathrm{p} 38$ on transcription factors was very dependent on the external cell environment. On the control surface, osterix was significantly reduced, Runx2 values were similar and p-Runx2 was significantly increased in comparison to osteogenic hASCs. On Ti flat osterix values were comparable, while Runx2/p-Runx2 was significantly increased. This positive outcome is in accordance with the enhanced extent of osteogenic markers. Even though Runx $2 / p$ Runx 2 values were similar and osterix was significantly increased on Ti lotus - with higher rates than on the control surface, but lower rates than on Ti flat - it still resulted in a reduced ALP activity with similar calcium production. Therefore, it can be concluded that the improved osteogenic differentiation after p38 inhibition is rather dependent on Runx2/p-Runx2 pathway (Figure 5 and Table 1).

On Ti flat and lotus higher rates of p-ERK and p-Smad and lower rates for ERK and BMP2 were obtained after ERK and JNK treatment when compared with the control surface. But comparing it with osteogenic hASCs, blocking of ERK and JNK caused an inhibition of ERK/p-ERK and BMP2/p-Smad pathway independently from the 
Citation: Fadeeva E, Deiwick A, Chichkov B, Schlie-Wolter S (2016) Enhanced Bioactivity of Titanium by Laser-generated Lotus-Topographies: Molecular Insights in Osteogenic Signaling Pathways of hASCs. J Nanomed Nanotechnol 7: 403. doi: 10.4172/2157-7439.1000403

Page 6 of 9

\begin{tabular}{|c|c|c|c|c|c|c|c|c|c|}
\hline \multicolumn{10}{|l|}{ Table 1A } \\
\hline Well & Osterix & Runx & pRunx & ERK $3 d$ & pERK & BMP2 & pSmad & ALP14 d & Ca $21 d$ \\
\hline Control & - - & -- & - - & - & - - & $=$ & - - & - - & - - \\
\hline DMSO & - & -- & -- & - & -- & $=$ & $=$ & $=$ & ++ \\
\hline ERK & - - - &.- & - - & +++ & - - & $=$ & - & - - & -- \\
\hline JNK & $\ldots$ & -- & -- & -- & $\ldots$ & -- & $\ldots$ & $\ldots$ & $\ldots$ \\
\hline p38 & $\cdots$ & $=$ & +++ & + & +++ &.- & - - & $=$ & +++ \\
\hline Ti flat & Osterix & Runx & pRunx & ERK $3 d$ & pERK & BMP2 & pSmad & ALP14 d & Ca $21 d$ \\
\hline DMSO & -- & + & + & + & + & $=$ & +++ & -- & + \\
\hline ERK & -- & $=$ & +++ & $--\cdot$ & $--\cdot$ & $=$ & -- & $--\cdot$ & - \\
\hline JNK & - - - & $=$ & $\ldots$ & $\ldots$ & - - - & - - - & - - & - - - & -- \\
\hline p38 & $=$ & +++ & +++ & - - & +++ & - - - & - & +++ & ++ \\
\hline Lotus & Osterix & Runx & pRunx & ERK 3d & pERK & BMP2 & pSmad & ALP14 d & Ca $21 d$ \\
\hline DMSO & $=$ & + & - & + & $=$ &.- & $=$ & - & + \\
\hline ERK & - - - &.- & - & - - & - - & - - - & - & - - - & - - - \\
\hline JNK & -- & $=$ & - & -- & -- & $=$ & -- & $--\cdot$ & -- \\
\hline p38 & +++ & $=$ & + & +++ & +++ & -- & - & - . - & $=$ \\
\hline \multicolumn{10}{|l|}{ Table 1B } \\
\hline Well-Ti flat & Osterix & Runx & pRunx & ERK $3 d$ & pERK & BMP2 & pSmad & ALP $14 d$ & Ca $21 d$ \\
\hline Osteo & +++ & +++ & +++ & +++ & +++ & $=$ & +++ & 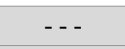 & ++ \\
\hline DMSO & +++ & +++ & +++ & +++ & +++ & $=$ & +++ &.- & ++ \\
\hline ERK & +++ & +++ & +++ & -. - & +++ & $=$ & +++ & - - & + \\
\hline JNK & +++ & +++ & +++ & $=$ & +++ & - & +++ & $\ldots$ & + \\
\hline p38 & +++ & +++ & +++ & - - & +++ & -- & +++ & $=$ & ++ \\
\hline Well-Lotus & Osterix & Runx & pRunx & ERK $3 d$ & pERK & BMP2 & pSmad & ALP $14 d$ & Ca $21 d$ \\
\hline Osteo & $=$ & +++ & +++ & - - & +++ & -- & $=$ & - & + \\
\hline DMSO & - - - & +++ & +++ & $=$ & +++ &.- & $=$ & - & + \\
\hline ERK & - - & +++ & +++ & - - - & +++ & - - - & $=$ & -. & - \\
\hline JNK & -- & +++ & +++ & - - & +++ & $=$ & ++ & - - - & + \\
\hline p38 & +++ & +++ & +++ & $=$ & +++ & $=$ & $=$ & - - & + \\
\hline Ti flat-Lotus & Osterix & Runx & pRunx & ERK $3 d$ & pERK & BMP2 & pSmad & ALP $14 d$ & Ca $21 d$ \\
\hline Osteo & - - & -- & - - & - - & - - & -- & - - - & + & $=$ \\
\hline DMSO & - - & -- & -- & -- & - - & -- & - - & ++ & - \\
\hline ERK & - - &.- & $\ldots$ &.- & - &.- & $\ldots$ & +++ & - \\
\hline JNK & - - - &.- & $=$ & - - & - - & $=$ & - - & + & - \\
\hline p38 &.- & $-\cdot$ & - - & + & $=$ & -- &.- & - - & -- \\
\hline
\end{tabular}

Table 1: Overview of the estimated correlation between lotus topography and insights in molecular osteogenic signaling pathway. (A) Effects of inhibitors and controls (control medium and DMSO solvent) in comparison to osteogenic hASCs. (B) Effects of the surface. No effects are signed by =; an increase with + , and decrease with -. Based on the Student's-t-test results with $p<0.05, p<0.01, p<0.001$, significant differences are highlighted. The results of the well plates refer to Supp. Figure 1 and Supp. Table 1 (p38 results on the control surface, ALP activity and calcium for all treatments) or to [18].

surface. The presence of $\mathrm{p} 38$ inhibitors also suppressed BMP2/p-Smad in an analogous manner, but the extent of suppression was less on $\mathrm{Ti}$ lotus followed by Ti flat. In all cases blocking of p38 caused a significant increase of ERK/p-ERK (Figures 6, 7 and Table 1)

This means that all three MAPKs crosstalk with these signaling cascades. Inhibition of ERK and JNK caused an inhibition of ERK/pERK and BMP2/p-Smad; inhibition of p38 inhibits BMP2/p-Smad, but activates ERK/p-ERK. This in turn has to correlate with the followed high extent of Runx2/p-Runx2. Even though Smads are formerly known to serve as a coactivator for Runx2 [18], their interaction after p38 blocking can be excluded on the control surface, since p-Smad values were reduced. This was not the case on Ti flat and lotus: higher rates of p-Smad and osterix occurred (Figures 5 and 7). However, the activated ERK/p-ERK cascade can explain why hASCs still undergo osteogenic differentiation after p38 blocking. In contrast to ERK and JNK the role of p38 was strongly dependent on the surface design. Therefore, it can be concluded that p38 plays a regulatory role in osteogenic differentiation, being dependent on the external cell environment. The strong attachment to surface, as seen for the lotustopography, supports it the best.

\section{Conclusion}

The molecular pathway of hASCs includes the MAPKs ERK, JNK, and p38 to stimulate osteogenic differentiation. They interact with ERK/p-ERK and BMP2/p-Smad signaling and activate the transcription factors osterix and Runx2/p-Runx2. ERK and JNK play a dominant role; their blocking abolishes all osteogenic markers. The functionality of p38 is regulatory and depends on the external cell environment. In this connection, the improved osteogenic differentiation on lotusstructures correlates with the enhanced cell attachment. This knowledge is essential to explain the improved bioactivity of laser-generated lotus structures - making this biomaterial functionalization very attractive for biomedical applications.

\section{Acknowledgements}

The authors thank Prof. A. Ngezahayo (Institute of Biophysics, Leibniz 
Citation: Fadeeva E, Deiwick A, Chichkov B, Schlie-Wolter S (2016) Enhanced Bioactivity of Titanium by Laser-generated Lotus-Topographies: Molecular Insights in Osteogenic Signaling Pathways of hASCs. J Nanomed Nanotechnol 7: 403. doi: 10.4172/2157-7439.1000403

A
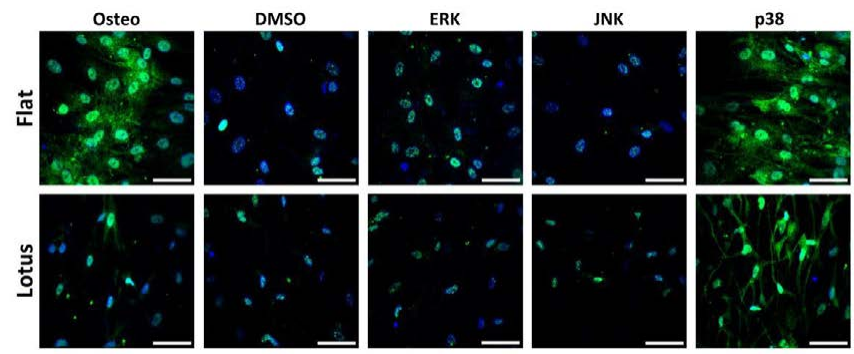

B

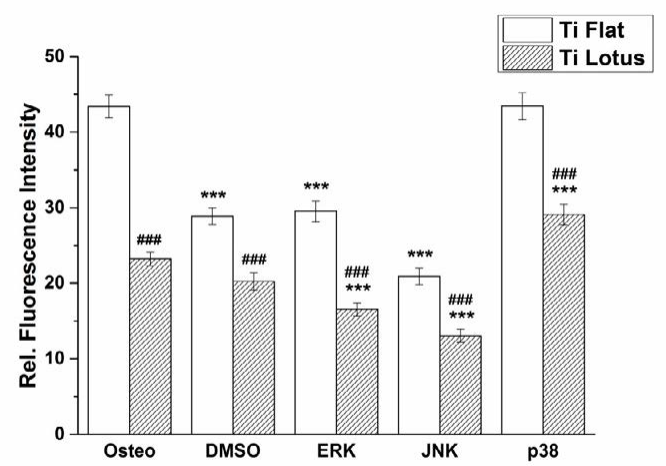

C
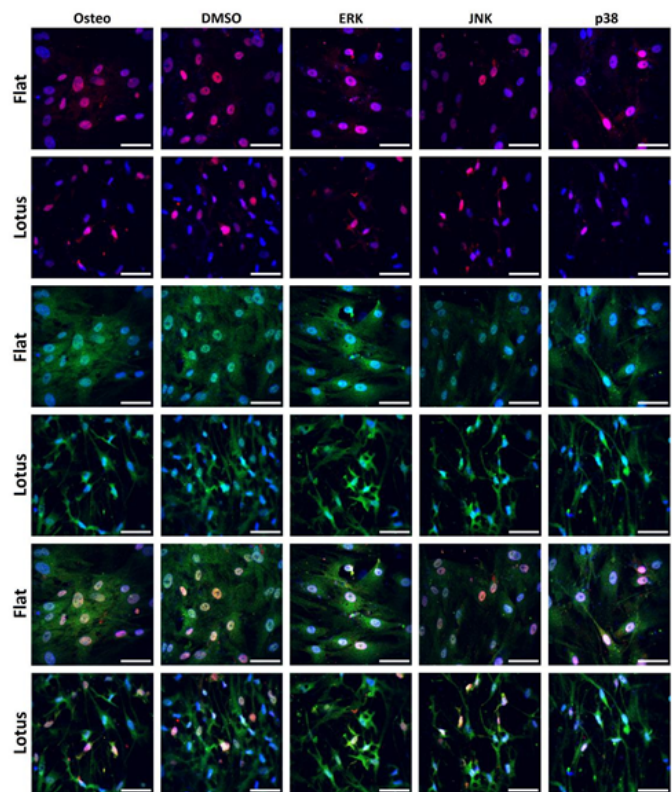

D

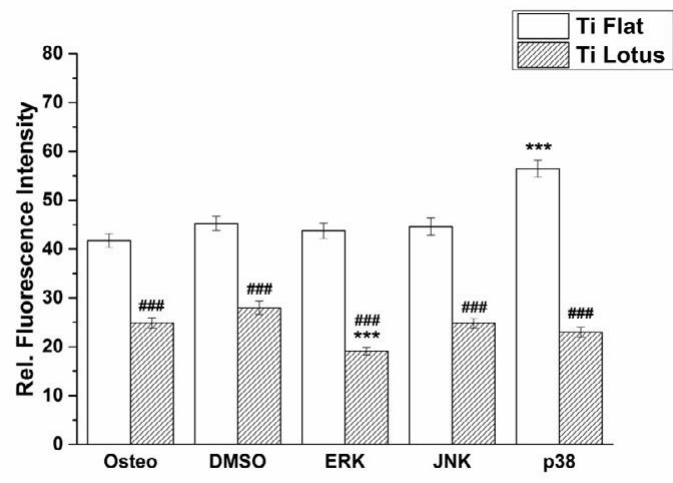

$\mathbf{E}$

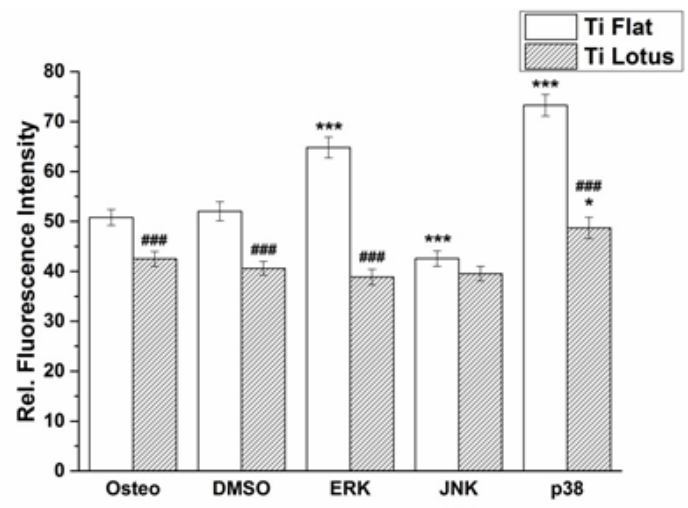

Figure 5: Analysis of the transcription factors after 3 days cultivation time. Fluorescence images of (A) osterix (green), and (C) Runx2 (red) and p-Runx2 (green). Nuclei (blue) are stained via Hoechst. Scale bars $=50 \mu \mathrm{m}$. Quantification of the relative fluorescence intensities obtained from 100 cells per treatment, given as average \pm SEM of (B) osterix, (D) Runx2, and (E) p-Runx2. Student's-t-test was applied $(p<0.05, p<0.01, p<0.001)$ to estimate significant differences between osteogenic and DMSO setup, and effects of the inhibitors of ERK, JNK, and p38 $\left(^{*}\right)$; and Ti flat with lotus topography (\#). 
A

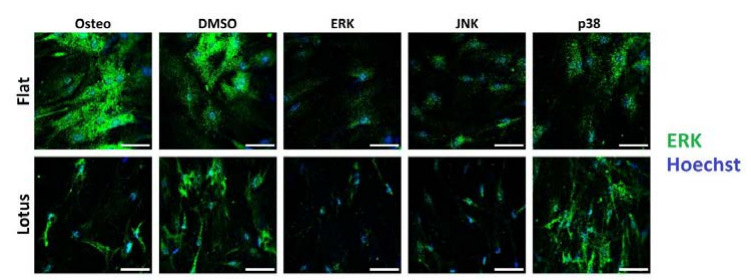

B

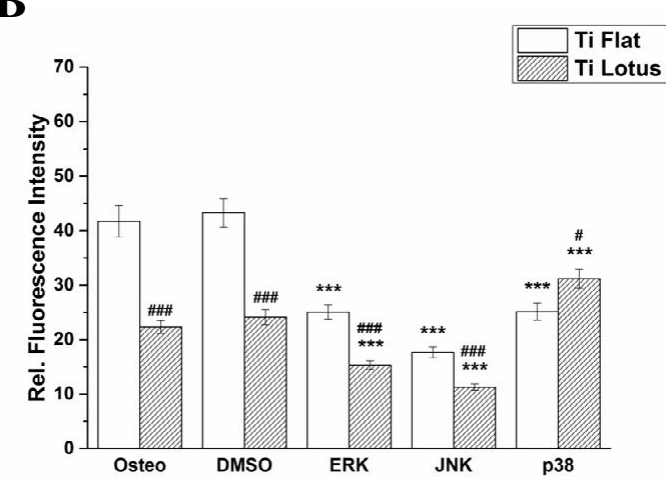

C

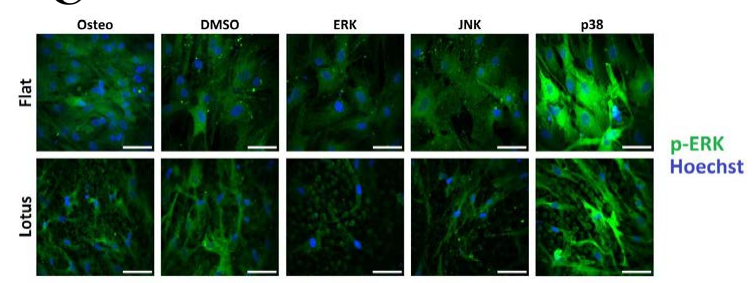

D

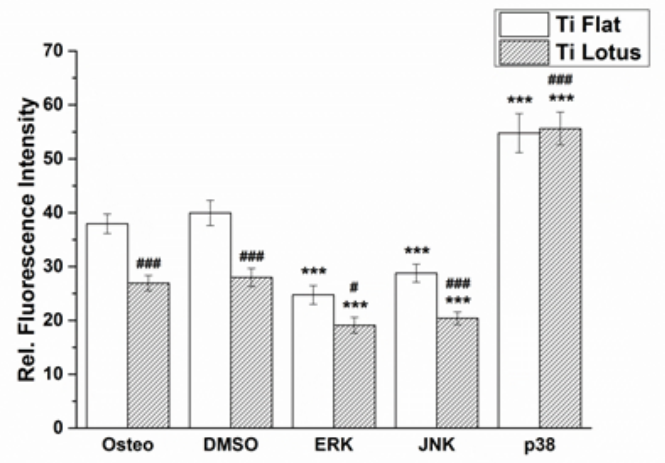

Figure 6: Analysis of ERK/p-ERK signaling cascade. Fluorescence images of (A) ERK (green), (C) p-ERK (green). Nuclei (blue) are stained via Hoechst. Scale bars $=50 \mu \mathrm{m}$. Quantification of the relative fluorescence intensities obtained from 100 cells per treatment, given as average $\pm S E M$ of $(B) E R K$, and $(D) p$-ERK. Student's-t-test was applied $(p<0.05, p<0.01, p<0.001)$ to estimate significant differences between osteogenic and DMSO setup, and effects of the inhibitors of ERK, $\mathrm{JNK}$, and $\mathrm{p} 38\left(^{*}\right)$; and Ti flat with lotus topography (\#).

A

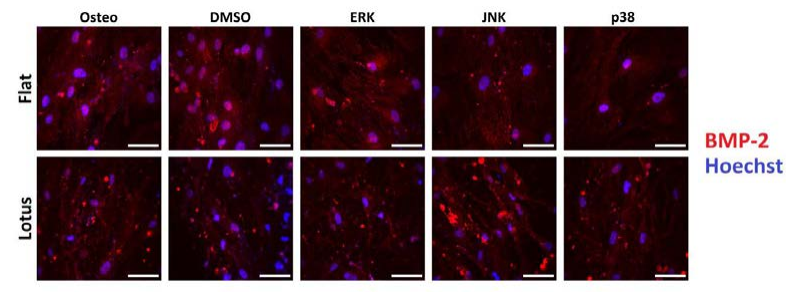

B

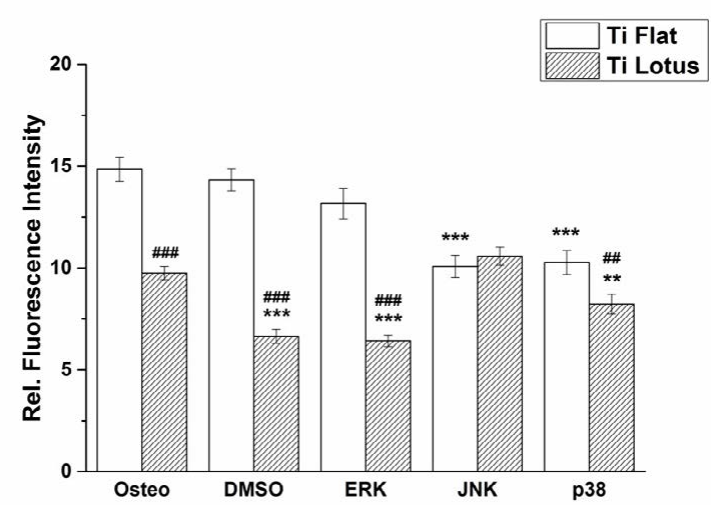

C

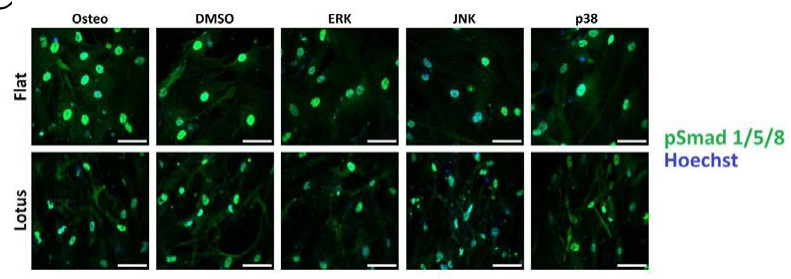

D

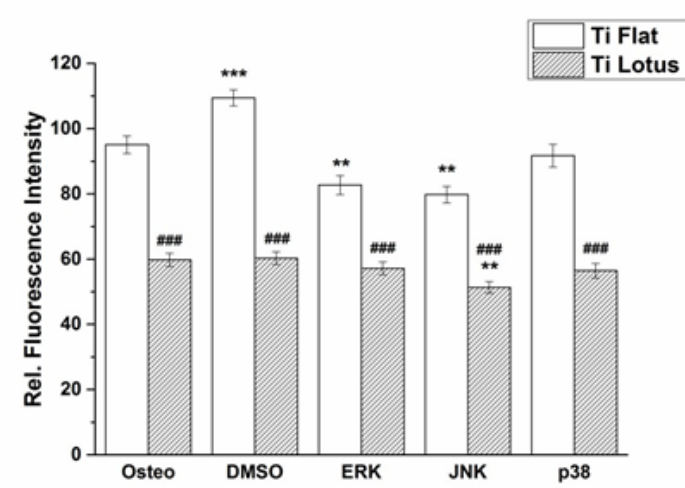

Figure 7: Analysis of BMP2/p-Smad signaling cascade. Fluorescence images of (A) BMP2 (red), (C) p-Smad 1/5/8 (green). Nuclei (blue) are stained via Hoechst. Scale bars $=50 \mu \mathrm{m}$. Quantification of the relative fluorescence intensities obtained from 100 cells per treatment, given as average \pm SEM of (C) BMP2, and (D) p-Smad 1/5/8. Student's-t-test was applied $(p<0.05, p<0.01, p<0.001)$ to estimate significant differences between osteogenic and DMSO setup, and effects of the inhibitors of ERK, JNK, and p38 $\left(^{*}\right)$; and Ti flat with lotus topography (\#). 
Citation: Fadeeva E, Deiwick A, Chichkov B, Schlie-Wolter S (2016) Enhanced Bioactivity of Titanium by Laser-generated Lotus-Topographies: Molecular Insights in Osteogenic Signaling Pathways of hASCs. J Nanomed Nanotechnol 7: 403. doi: 10.4172/2157-7439.1000403

University of Hannover, Germany) for granting the use of fluorescence microscope. This work was partly supported by "Biofabrication for NIFE" and Cluster of Excellence "Rebirth". Dr. S. Schlie-Wolter thanks for her Habilitation scholarship of the Leibniz University of Hannover, Germany.

\section{References}

1. Schuh JCA (2008) Medical device regulations and testing for toxicologic pathologists. Toxicologic Pathology 36: 63-69.

2. Geetha M, Singh AK, Asokamani R, Gogia AK (2009) Ti based biomaterials, the ultimate choice for orthopeadic implants - a review. Prog Mater Sci 54: 397-425.

3. Jones JA (2006) Observing cell responses to biomaterials. Materials Today 9: $34-43$.

4. Bosco R, Van den Beucken J, Leeuwenburgh S, Jansen J (2012) Surface engineering for bone implants: a trend from passive to active surfaces. Coatings 2: 95-119.

5. Triplett RG, Andrews JA, Hallmon WW (2003) Management of peri-implantitis Oral Maxillofacial Surg Clin N Am 15: 129-138.

6. Fadeeva E, Deiwick A, Chichkov B, Schlie-Wolter S (2013) Impact of laser structured biomaterial interfaces on guided cell responses. Interface Focus.

7. Barradas AMC, Yuan H, van Blitterswijk CA, Habibovic $P$ (2011) Osteoinductive biomaterials: current knowledge of properties, experimental models and biological mechanism. Eur Cells Mater 21: 407-429.

8. Dohan Ehrenfest DM, Coelho PG, Kang BS, Sul YT, Albrektsson T (2009) Classification of osseointegrated implant surfaces: materials, chemistry and topography. Trend Biotechnol 28: 198-206.

9. Schlie-Wolter S, Ngezahayo A, Chichkov B (2013) The selective role of extracellular matrix components on cell adhesion, morphology, proliferation and communication in vitro. Exp Cell Research 319: 1553-1561.

10. Barrère F, Mahmood TA, de Groot K, Blitterswijk CA (2008) Advanced biomaterials for skeletal tissue regeneration: instructive and smart functions. Mater Scie Eng 59: 38-71.

11. Flemming RG, Murphy CJ, Abrams GA, Goodman SL (1999) Effects of synthetic micro- and nano-structured surfaces on cell behaviour. Biomaterials 20: $573-588$

12. Liu H, Webster TJ, Liu H (2007) Nanomedicine for implants: a review of studies and experimental tools. Biomaterials 28: 354-369.

13. Schlie S, Fadeeva E, Koch J, Ngezahayo A, Chichkov BN (2010) Femtosecond laser fabricated spike structures for selective control of cellular behavior. J Biomater Appl 25: 217-233.
14. Schlie S, Fadeeva E, Koroleva A, Ovsianikov A, Koch J, et al. (2011) Laserbased nanoengineering for biomedical applications. Photonic Nanostruct 9 . $159-162$.

15. Fadeeva E, Truong VK, Stiesch M, Chichkov BN, Crawford RJ, et al. (2012) Bacterial retention on superhydrophobic titanium surfaces by femtosecond laser ablation. Langmuir 26: 3012-3019.

16. Schlie-Wolter S, Deiwick A, Fadeeva E, Paasche G, Lenarz T, et al. (2013) Topography and coating of platinum improve the electrochemical properties and neuronal guidance. Applied Materials and Interfaces 5: 1070-1077.

17. Deiwick A, Fadeeva E, Koch L, Gebauer R, Chichkov B, et al. (2014) Functional titanium lotus-topography promotest he osteoinduction of human adiposederived stem cells in vitro. J Nanomed Nanotechnol 5:239.

18. Deiwick A, Schlie-Wolter S (2016) Osteogenic differentiation of human adiposederived stem cells (hASCs): role of FAK and Ras-MAPK signaling. Journal of Stem Cell and Regenerative Biology.

19. Schindeler A, Little DG (2006) Ras-MAPK signaling in osteogenic differentiation friend or foe? J Bone Miner Res 21: 1331-1338.

20. Engler AJ, Sen S, Sweeney HL, Discher DE (2006) Matrix elasticity directs stem cell lineage specification. Cell 126: 677-689.

21. Dellatore SM, Garcia AS, Miller WM (2008) Mimicking stem cell niches to increase stem cell expansion. Curr Opin Biotechnol 19: 534-540.

22. McNamara LE, Murray RJ, Biggs MJP, Kentawong F, Oreffo ROC, et al. (2010) Nanotopographical control of stem cell differentiation. J Tissue Eng

23. Asatrian G, Pham D, Hardy WR (2008) Stem cell technology for bone regeneration: current status and potential applications. Stem Cells Cloning 8 : $39-48$

24. Birmingham E, Niebur GL, McHugh PE, Shaw G, Barry FP, et al. (2012) Osteogenic differentiation of mesenchymal stem cells is regulated by osteocyte and osteoblast cells in a simplified bone niche. Eur Cells Mater 23: 13- 27.

25. Artigas N, Urena C, Rodríguez-Carballo E, Rosa JL, Ventura F (2014) Mitogenactivated protein kinase (MAPK)-regulated interactions between osterix and Runx2 are critical for the transcriptional osteogenic program. J Biol Chem 289: 27105-27117.

26. Hirano T, Sasaki H, Honma S, Furuya Y, Miura T, et al. (2015) Proliferation and osteogenic differentiation of human mesenchymal stem cells on zirconia and titanium with different surface topography. Dent Mater J 34: 872-880.

27. Vinals F, Lopez-Rovira T, Rosa JL, Ventura F (2002) Inhibition of PI3K/p70 S6K and p38 MAPK cascades increases osteoblastic differentiation induced by BMP-2. FEBS letters 510: 99-104. 